\title{
Comparative Evaluation of the Pathloss Prediction Performance Hata-Okumura Pathloss Model for Urban, Suburban and Rural Areas
}

\author{
Steve Worgu, Samuel Godwin Ajumo, Njumoke N. Odu \\ Department of Electrical/Computer Engineering, Port Harcourt Polytechnic, Rumuola, Port Harcourt, Nigeria
}

Email address:

stevewa438@yahoo.com (S. Worgu)

\section{To cite this article:}

Steve Worgu, Samuel Godwin Ajumo, Njumoke N. Odu. Comparative Evaluation of the Pathloss Prediction Performance Hata-Okumura Pathloss Model for Urban, Suburban and Rural Areas. International Journal of Systems Science and Applied Mathematics.

Vol. 2, No. 1, 2017, pp. 42-50. doi: 10.11648/j.jjssam.20170201.16

Received: October 31, 2016; Accepted: January 5, 2017; Published: February 23, 2017

\begin{abstract}
In this paper, comparative evaluation of the pathloss prediction performance of the popular Hata-Okumura model for urban, suburban and rural areas is carried out. The study is based on empirical measurements conducted at Imo state university campus for $800 \mathrm{MHz}$ GSM network. The prediction performance of the three categories of the Hata-Okumura model is analyzed and compared in terms of Mean Absolute Error (MAE), Root Mean Square Error (RMSE), and Prediction Accuracy (PA). The results showed that the Hata-Okumura model for the Urban area has the best prediction accuracy when not optimised but it has the lowest optimised prediction accuracy. On the other hand the Hata-Okumura model for the rural/open area has the least prediction accuracy when not optimised but it has the highest prediction accuracy when it is optimised. The results show that adoption of a model because it has the highest prediction accuracy is not the best approach to the selection of pathloss models. Rather the models should be optimised and the best optimised model should be adopted.
\end{abstract}

Keywords: Pathloss, Hata-Okumura Model, Pathloss Model, Model Tuning, Least Square Method

\section{Background of the Study}

In the telecommunication industry, pathloss is the reduction in power density of an electromagnetic wave signal at it propagates from the transmitter to the receiver [1]. Accurate prediction of pathloss is very essential in GSM network planning and optimization. Propagation pathloss models are used to calculate pathloss during transmission of a signal so as to predict the mean signal strength for an arbitrary transmitter-receiver separation distance [2, 3, 4, 5]. In general, pathloss models are categorized as empirical, stochastic and deterministic [6, 8]. Among the three categories, the empirical models are frequently used for outdoor pathloss predictions. However, in practice, empirical pathloss model tuning is usually required due to significant drop in prediction performance of empirical models when applied in the environments other than the ones they are designed.

Generally, the goal of model tuning is to minimize the difference between measured pathloss and corresponding model predicted pathloss [8]. The tuning can be done by adding correction factor to the original model or by modifying the coefficients of some of the model's parameters. Among the different model tuning approaches, the Root Mean Square Error (RMSE) based approach has been the easiest and most popular. In the RMSE-based tuning approach, the RMSE between the predicted and the measured pathloss is used as the correction factor which is either added or subtracted from the model to minimize its prediction error. After tuning, the pathloss model prediction performance is evaluated using statistical parameters such as the RMSE, the coefficient of determination $\left(R^{2}\right)$, among others. According to available literatures, the performance of a pathloss model is considered acceptable if it provides an overall RMSE of about 6-7dB for urban areas and 10 to $15 \mathrm{~dB}$ for suburban and rural areas [26, 27, 28]. Studies have also shown that most empirical pathloss models have high prediction errors with RMSE above the given acceptable range for the particular environment being studied. Therefore, model tuning is usually employed to reduce the model prediction error so that the RMSE falls within the acceptable range.

In most published works on empirical pathloss models, usually, different pathloss models are studied and compared 
in terms of their pathloss prediction performance and the model with best prediction performance is select and then optimized for the study area. In this paper, the Hata Okumura pathloss model is studied. Specifically, the three categories of the Hata-Okumura model are employed in the pathloss prediction for a university campus. The pathloss prediction performance of the models are compared before and after optimization. The study seek to establish that in some cases, models that has the best prediction performance before optimisation may not give the best prediction after optimisation.
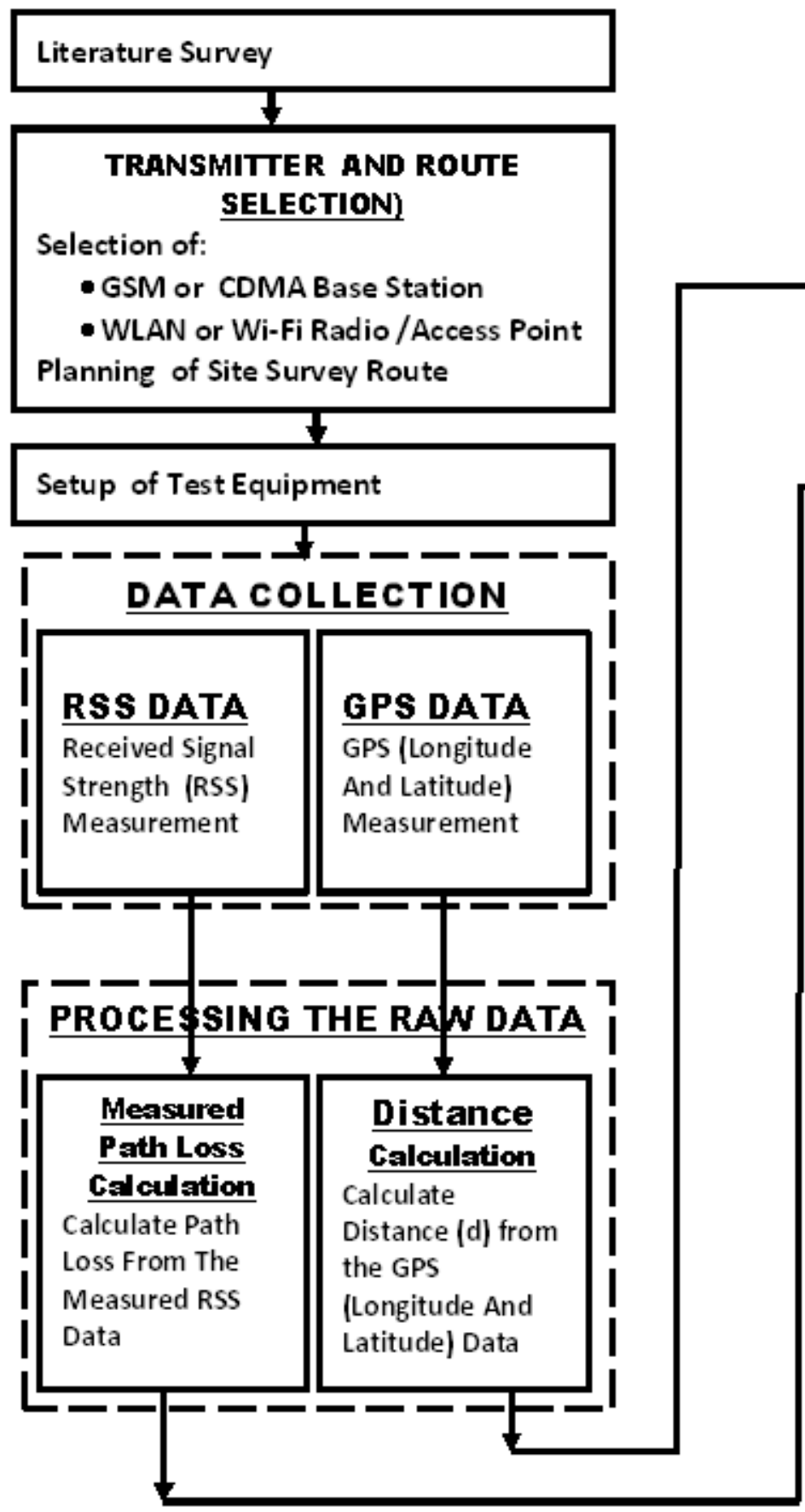

Figure 1. The Research Methodology.

\section{The Methodology}

The methodology that is used to accomplish the objectives of the paper is given in Figure 1. The research method is a combination of empirical and simulation approaches. According to Figure 1, the work started with literature review of relevant studies along with the review of published works on the propagation environments that are considered in the paper. The research method can be applied for optimisation of any empirical pathloss model based on data captured from drive test on wireless networks which can be Wi-Fi or GSM networks.

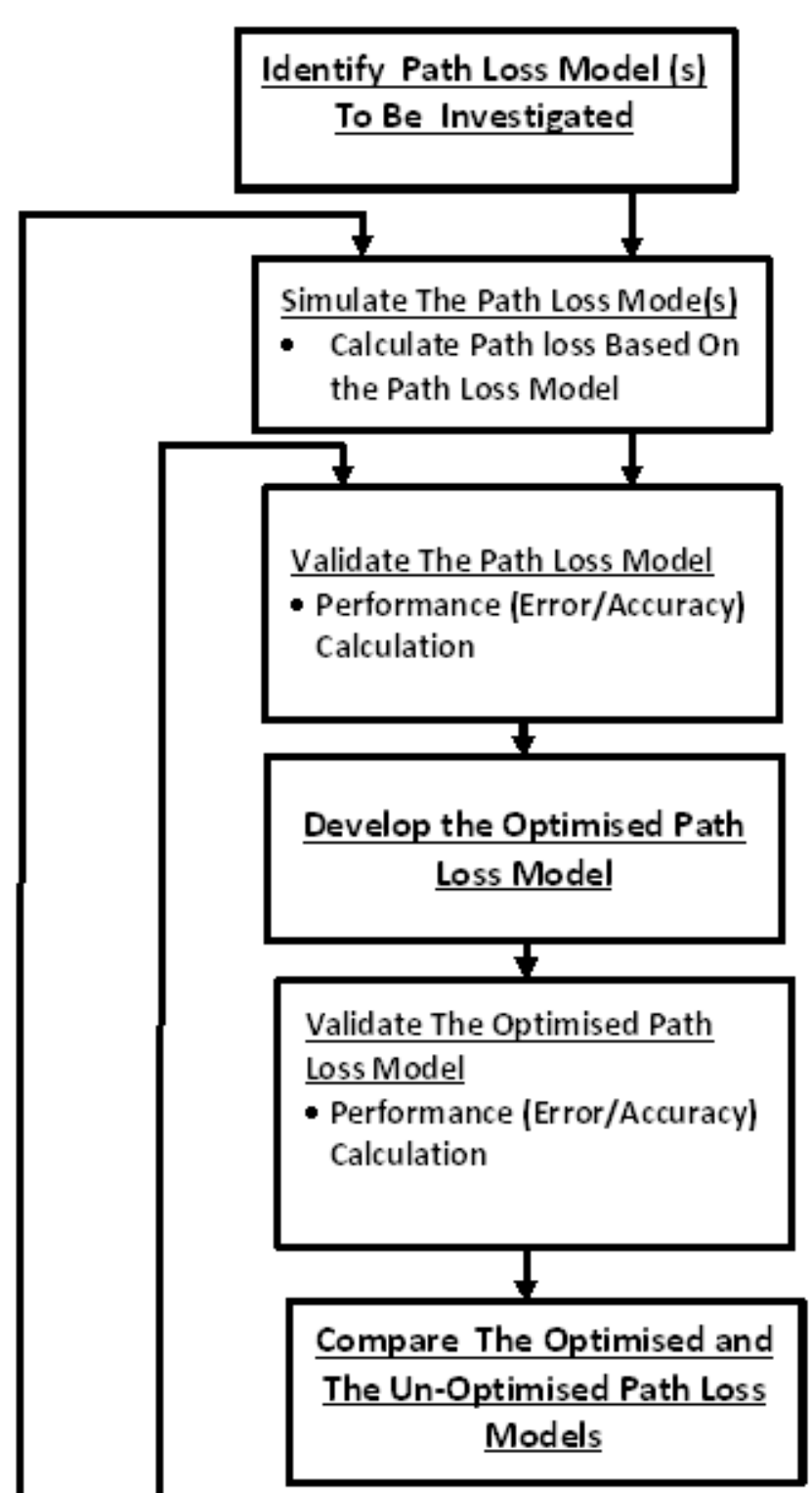


Among other data collected during the measurement campaign, the Received Signal Strength (RSS) and spatial data (longitude and latitude) are further processed to obtain the measured pathloss (PL) and the transmission distance (d) for each of the locations where data is collected. The distance (d) data is used to simulate the selected pathloss model to generate the predicted pathloss. The prediction accuracy of the pathloss model is evaluated with respect to the measured pathloss. The pathloss model is then optimised to improve on the prediction accuracy of the selected pathloss model. The optimised pathloss model is validated by evaluating its prediction accuracy. Finally, the prediction accuracy of the optimised pathloss model is compared with the prediction accuracy of the original un-optimised pathloss model. Particularly, in this paper, the urban, suburban and rural versions of the popular Hata-Okumura pathloss model are studied and compared for their pathloss prediction performance for a university campus in Imo state, Nigeria

\subsection{Okumura Pathloss Model}

Hata-Okumura pathloss model is derived from the original graphical information presented in Okumura Model [12, 13, $14,15]$ The basic Hata-Okumura model is for urban areas and it is a widely used propagation model for predicting path loss in urban areas. This model takes into account the effects of diffraction, reflection and scattering caused by city structures. Hata-Okumura model has specific model versions for urban, suburban and rural areas.

The Okumura's model is applicable to frequencies within the range of 150 to $1920 \mathrm{MHz}$, transmitter height of $30 \mathrm{~m}$ to $200 \mathrm{~m}$, Link Distance of $1 \mathrm{~km}$ to $20 \mathrm{~km}$, Mobile Station (MS) height of $1 \mathrm{~m}$ to $10 \mathrm{~m}$. The pathloss prediction formula according to Okumura's model is expressed as follows [13, 14, $15,16]$ :

$$
\begin{aligned}
& L P_{O_{K_{-}} H A T A(u r b a n)}=A+B * \log _{10}(d) \text { for Urban Area } \\
& L P_{O_{K} H_{A T A}(\text { suburban })}=A+B * \log _{10}(d)-C \text { for Suburban Area } \\
& L P_{O K_{-} H A T A(o p e n / \text { rural })}=A+B * \log _{10}(d)-D \text { for Open Area } / \text { Rural } \\
& A=69.55+26.16 * \log _{10}(f)-13.82 * \log _{10}\left(h_{b}\right)-a\left(h_{m}\right) \\
& B=44.9-6.55 * \log _{10}\left(h_{b}\right) \\
& C=5.4+2 *\left[\log _{10}\left(\frac{f}{28}\right)\right]^{2} \\
& D=40.94+4.78 *\left[\log _{10}(f)\right]^{2}-18.33 * \log _{10}(f) \\
& a\left(h_{m}\right)=\left[1.1 * \log _{10}(f)-0.7\right] * h_{m}-\left[1.56 * \log _{10}(f)-0.8\right]
\end{aligned}
$$

Eq 3.8 is for small city, medium city, open area, rural area and sububan area

$$
\begin{aligned}
& a\left(h_{m}\right)=8.28 *\left[\log _{10}\left(1.54 * h_{m}\right)\right]^{2}-1.1 \text { for large city } \mathrm{f} \leq 200 \mathrm{MHz} \\
& a\left(h_{m}\right)=3.2 *\left[\log _{10}\left(11.75 * h_{m}\right)\right]^{2}-4.97 \text { for large city } \mathrm{f} \geq 400 \mathrm{MHz}
\end{aligned}
$$

Where

- $\mathrm{f}$ is the centre frequency $\mathrm{f}$ in $\mathrm{MHz}$

- $\mathrm{d}$ is the link distance in $\mathrm{km}$

- $a\left(h_{m}\right)$ is an antenna height-gain correction factor that depends upon the environment

- $\mathrm{C}$ and D are used to correct the small city formula for suburban and open areas

- $150 \mathrm{MHz} \leq \mathrm{f} \leq 1000 \mathrm{MHz}$

- $30 \mathrm{~m} \leq h_{b} \leq 200 \mathrm{~m}$

- $1 \mathrm{~m} \leq h_{m} \leq 10 \mathrm{~m}$

- $1 \mathrm{~km} \leq \mathrm{d} \leq 20 \mathrm{~km}$

\subsection{Data Collection}

The measurement campaign is carried out in Imo state university campus for a GSM network operating in the $800 \mathrm{MHz}$ frequency band. A drive test was conducted to obtain the actual field measurement data which was later used in tuning and appraising the accuracy of the empirical pathloss models under study. The drive test equipment consists of Android/Smart mobile phone which hosts and runs all the software used for capturing the GPS data as well as the RSS data in $\mathrm{dB}$. Particularly, Cellmapper android application was installed on the android phone used for the measurement campaign. The cellmapper application captures and logs the received signal strength (RSS), the cell tower data, GPS data of the measurement point, among others. The data is exported as text of CSV file which is then used for the pathloss analysis.

\subsection{Calculation of the Measured Path Length from the GPS Coordinates}

The GPS coordinates captured for each measurement point are used to determine the distance of the measurement point from the transmitting station. Haversine formula Eq 11 is used to computer the distances or path lengths as follows; 


$$
d=2 r\left\{\sqrt[2]{\sin \left(\frac{L A T_{2}-L A T_{1}}{2}\right)^{2}+\cos \left(L A T_{1}\right) \cos \left(L A T_{2}\right) \sin \left(\frac{L O N G_{2}-L O N G_{1}}{2}\right)^{2}}\right\}
$$

LAT in Radians $=\frac{(\text { LAT in Degrees } * 3.142)}{180}$

$$
\text { LONG in Radians }=\frac{(\text { LONG in Degrees } * 3.142)}{180}
$$

Where

LAT 1 and LAT2 are the latitude of the coordinates of point 1 and point 2 respectively

LONG1 and LONG2 are the longitude of the coordinates of point 1 and point 2 respectively

$\mathrm{R}=$ radius of the earth $=6371 \mathrm{~km}$

$\mathrm{d}=$ the distance between the two coordinates

$\mathrm{R}$ varies from $6356.752 \mathrm{~km}$ at the poles to $6378.137 \mathrm{~km}$ at the equator

\subsection{Calculation of the Measured Pathloss from the Measured RSS}

Each of the Received Signal Strength (RSS) value recorded at various distances from each of the routes for the GSM base station is converted to Measured Pathloss $\left(P L_{m(d B)}\right)$ using the formula (Ajose and Imoize, 2013; Rappaport 2002; Seybold 2005):

Pathloss Measured $=P L_{m(d B)}=\mathrm{EIRP}_{\mathrm{t}}(\mathrm{dBm})-\mathrm{P}_{\mathrm{r}}(\mathrm{dBm})(14)$

where $P L_{m(d B)}$ is the measured pathloss for each measurement location at a distance $\mathrm{d}(\mathrm{km})$, EIRPt is the Effective Isotropic Radiated Power in $\mathrm{dBm}$ and $\mathrm{Pr}$ is the mean Received Signal Strength (RSS) in $\mathrm{dBm}=$ the measured received signal strength.

The effective isotropic radiated power EIRPt $(\mathrm{dBm})$ is given as:

$$
\mathrm{EIRPt}=\mathrm{PBTS}+\mathrm{GBTS}+\mathrm{GMS}-\mathrm{LFC}-\mathrm{LAB}-\mathrm{LCF}
$$

where PBTS $=$ Transmitter Power $(\mathrm{dBm})$, GBTS = Transmitter Antenna Gain $(\mathrm{dBi}), \mathrm{GMS}=$ receiver antenna gain $(\mathrm{dBi}), \mathrm{LFC}=$ feeder cable and connector loss $(\mathrm{dB}), \mathrm{LAB}$ $=$ Antenna Body Loss $(\mathrm{dB})$ and $\mathrm{LCF}=$ Combiner and Filter Loss $(\mathrm{dB})$. The values of these parameters are given as (Ajose and Imoize, 2013; Mishra, 2007; Saunders and Aragon-Zavala, 2007) as: $\mathrm{PBTS}=40 \mathrm{~W}=[30+10 \log 1040]=46 \mathrm{dBm}$, $\mathrm{GBTS}=16 \mathrm{dBd}=[16+2.15]=18.15 \mathrm{dBi}, \mathrm{GMS}=0 \mathrm{dBi}, \mathrm{LFC}$ $=3 \mathrm{~dB}, \mathrm{LAB}=3 \mathrm{~dB}$, and $\mathrm{LCF}=4.7 \mathrm{~dB}$. Substituting these values into equation (11) gives; EIRPt $=46+18.15-3-3-$ 4.7 $\mathrm{EIRPt}=53.5 \mathrm{dBm}$.

The pathloss values measured in $\mathrm{dB}$ are obtained by substituting the calculated value of EIRPt $(\mathrm{dBm})$ and the measured values of $\operatorname{Pr}(\mathrm{dBm})$ into equation 10 .

\subsection{Performance Analysis of the Models}

In this paper, prediction performance of the pathloss models are evaluated in terms of Mean Absolute Error (MAE), Root Mean Square Error (RMSE), and Prediction Accuracy (PA).

The Mean Absolute Error (MAE) between the measured data and the theoretical estimations is calculated as follows:

$$
\operatorname{MAE}=\frac{1}{n}\left(\sum_{i=1}^{i=n}\left|P L_{(\text {measured })(i)}-P L_{(\text {predicted })(i)}\right|\right)
$$

where: $P L_{(\text {measured })(i)}$ is the measured pathloss $(\mathrm{dB})$, $P L_{(\text {predicted })(i)}$ is the predicted pathloss $(\mathrm{dB})$, and $\mathrm{n}$ is the number of measured data points.

The Root Mean Square Error (RMSE) is calculated as follows:

$$
\begin{gathered}
\text { MSE }=\sqrt[2]{\left\{\frac{1}{n}\left[\sum_{i=1}^{i=n}\left|P L_{(\text {measured })(i)}-P L_{(\text {predicted })(i)}\right|^{2}\right]\right\}} \\
\text { Square Of Error }=\left|P L_{(\text {measured })(i)}-P L_{(\text {predicted })(i)}\right|^{2} \\
\therefore \text { MSE }=\sqrt[2]{\left\{\frac{1}{n}\left[\sum_{i=1}^{i=n}(\text { Square Of Error })\right]\right\}}
\end{gathered}
$$

where: $P L_{(\text {measured })(i)}$ is the measured pathloss $(\mathrm{dB})$, $P L_{(\text {predicted })(i)}$ is the predicted pathloss $(\mathrm{dB}), \overline{P L_{(\text {measured })}}$ is the mean of measured pathloss, and $n$ is the number of measured data points.

The prediction accuracy (PA, \%) based on mean absolute percentage deviation (MAPD) or Mean Absolute Percentage Error (MAPE) is calculated as follows:

$$
\mathrm{PA}=\left\{1-\frac{1}{n}\left(\sum_{i=1}^{i=n}\left|\frac{\left|P L_{(\text {measured })(i)}-P L_{(\text {predicted })(i)}\right|}{P L_{(\text {measured })(i)}}\right|\right)\right\} * 100 \%
$$

\subsection{Model Optimization}

Pathloss model optimization is a process in which a theoretical propagation model is adjusted with the help of measured values obtained from test field data. The aim is to get the predicted field strength as close as possible to the measured field strength. The parameters of the Hata-Okumura pathloss models are adjusted (optimized) using least square algorithm. The simple approach to accomplish the tuning is as follows;

- Compute the Sum of errors and the RMSE from the measured and the model predicted pathloss values. In this case, the error computed as $P L_{(\text {measured })(i)}-$ $P L_{(\text {predicted })(i)}$.

- Next, the RMSE is added to each predicted pathloss value if the sum of error is positive; otherwise, the RMSE is subtracted from each predicted pathloss value. The optimized model is therefore, the original model plus the RMSE. The pathloss prediction performance of the optimized model is then determined and compared with untuned

\section{Results and Discussion}

Measured Received Signal Strength (RSSI), Measured Pathloss and Distance for GSM operating in the $800 \mathrm{MHz}$ 
frequency band are given in Table 1. The GSM base station longitude and latitude are 7.047507 and 5.510168 respectively. The base station coordinates and the coordinates of each of the measurement point are used in a Harversine formula to determine distance of each of the measurement point from the base station. The measured RSSI values were converted to measured Pathloss using Eq 7 and Eq 8. Figure 2, give the radial graph of measured RSS $(\mathrm{dB})$ and measured path loss $(\mathrm{dBm})$.

Table 1. The Measured Received Signal Strength (RSSI) and Measured Pathloss and Distance.

\begin{tabular}{|c|c|c|c|c|c|c|}
\hline $\mathbf{S} / \mathbf{N}$ & Longitude & Latitude & d (km) & f (MHz) & RSSI (dBm) & Field Measured Path Loss (dBm) \\
\hline 1 & 7.040935 & 5.507852 & 0.7726 & 800 & -81 & 106.3 \\
\hline 2 & 7.040672 & 5.507752 & 0.8038 & 800 & -85 & 110.3 \\
\hline 3 & 7.040565 & 5.50762 & 0.8199 & 800 & -85 & 110.3 \\
\hline 5 & 7.040467 & 5.507362 & 0.8404 & 800 & -85 & 110.3 \\
\hline 6 & 7.040458 & 5.507215 & 0.8475 & 800 & -85 & 110.3 \\
\hline 7 & 7.040433 & 5.507063 & 0.8568 & 800 & -91 & 116.3 \\
\hline 8 & 7.040448 & 5.506895 & 0.863 & 800 & -91 & 116.3 \\
\hline 9 & 7.041365 & 5.5054 & 0.8632 & 800 & -91 & 116.3 \\
\hline 10 & 7.040448 & 5.506722 & 0.8713 & 800 & -91 & 116.3 \\
\hline 11 & 7.041168 & 5.505432 & 0.8784 & 800 & -91 & 116.3 \\
\hline 12 & 7.040332 & 5.50657 & 0.8903 & 800 & -91 & 116.3 \\
\hline 13 & 7.040413 & 5.506352 & 0.8936 & 800 & -91 & 116.3 \\
\hline 14 & 7.040918 & 5.50544 & 0.9001 & 800 & -91 & 116.3 \\
\hline 15 & 7.040405 & 5.50624 & 0.9004 & 800 & -97 & 122.3 \\
\hline 16 & 7.040135 & 5.506553 & 0.9107 & 800 & -97 & 122.3 \\
\hline 17 & 7.040382 & 5.506077 & 0.9115 & 800 & -97 & 122.3 \\
\hline 19 & 7.039983 & 5.506675 & 0.92 & 800 & -97 & 122.3 \\
\hline 20 & 7.040647 & 5.50549 & 0.9215 & 800 & -97 & 122.3 \\
\hline 21 & 7.040435 & 5.505672 & 0.9299 & 800 & -97 & 122.3 \\
\hline 22 & 7.039803 & 5.506722 & 0.936 & 800 & -97 & 122.3 \\
\hline 23 & 7.03963 & 5.50678 & 0.9509 & 800 & -97 & 122.3 \\
\hline 24 & 7.039477 & 5.506815 & 0.965 & 800 & -97 & 122.3 \\
\hline 25 & 7.039288 & 5.50683 & 0.9837 & 800 & -97 & 122.3 \\
\hline 26 & 7.03911 & 5.506847 & 1.0013 & 800 & -97 & 122.3 \\
\hline 27 & 7.039023 & 5.506815 & 1.0116 & 800 & -99 & 124.3 \\
\hline
\end{tabular}

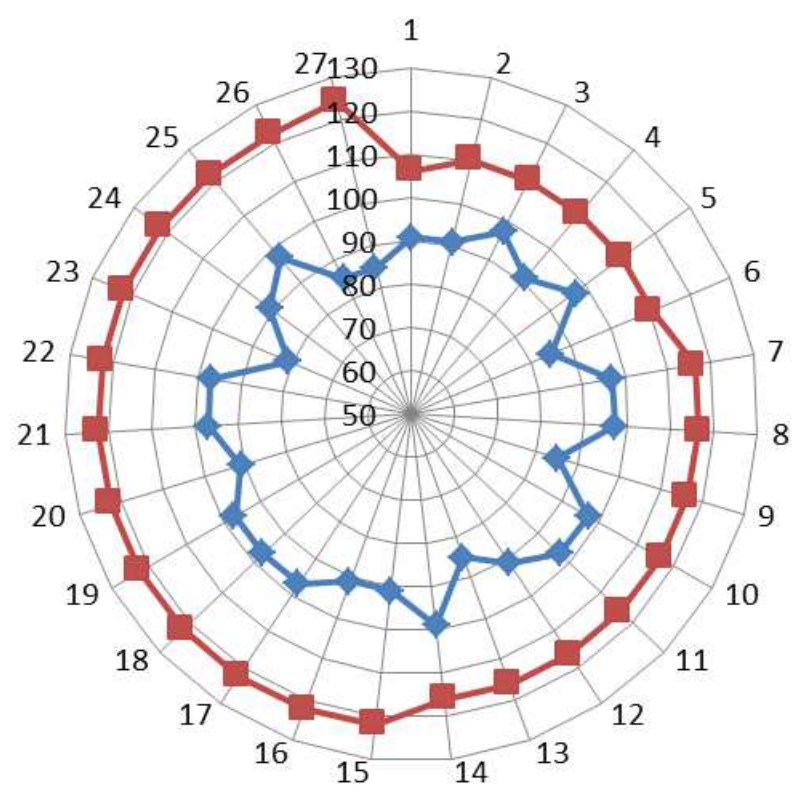

Figure 2. The Radial Graph Of Measured RSS $(d B)$ and Measured Path Loss (dBm) vs Distance.
Table 2 and figure 3 show the predicted pathloss $(\mathrm{dB})$ versus distance for the un-tuned Hata-Okumura Models. Also, Table 3 and figure 4 show the predicted pathloss $(\mathrm{dB})$ versus distance for the optimised Hata-Okumura Models. Table 4 and figure 5 give the prediction performance of the models in terms of RMSE value. Table 5 and figure 6 give the prediction performance of the models in terms of Mean Absolute Error (MAE) whereas Table 6 and figure 7 give the prediction performance of the models in terms of prediction accuracy (\%).

From the table 4, 5 and 6 and bar charts of figure 5, 6 and 7, it can be stated that the Hata-Okumura model for the urban area has the best prediction accuracy when not optimised but it has the lowest prediction accuracy when optimised. On the other hand, the Hata-Okumura model for the rural/open area has the least prediction accuracy when not optimised but it has the highest prediction accuracy when it is optimised. The improvement in the prediction accuracy for the three models are: Hata-Okumura Urban (8.49\%), Hata-Okumura Sub-Urban (16.73\%) and Hata-Okumura Rural/Open $(29.91 \%)$.

Table 2. Predicted Pathloss (dB) vs Distance For The Un-tuned Hata-Okumura Models.

\begin{tabular}{lllll}
\hline $\mathbf{d}(\mathbf{k m})$ & Field Measured Pathloss $(\mathbf{d B m})$ & Hata-Okumura Urban & Hata-Okumura Suburban & Hata-Okumura Open /RURAL AREA \\
\hline 0.7726 & 106.3 & 15.08 & 24.34 & 39.57 \\
0.8038 & 110.3 & 15.08 & 24.34 & 39.57 \\
0.8199 & 110.3 & 15.08 & 24.34 & 39.57 \\
0.8297 & 110.3 & 15.08 & 24.34 & 39.57 \\
\hline
\end{tabular}




\begin{tabular}{lllll}
\hline $\mathbf{d}(\mathbf{k m})$ & Field Measured Pathloss $\mathbf{( d B m})$ & Hata-Okumura Urban & Hata-Okumura Suburban & Hata-Okumura Open /RURAL AREA \\
\hline 0.8404 & 110.3 & 15.08 & 24.34 & 39.57 \\
0.8475 & 110.3 & 15.08 & 24.34 & 39.57 \\
0.8568 & 116.3 & 15.08 & 24.34 & 39.57 \\
0.863 & 116.3 & 15.08 & 24.34 & 39.57 \\
0.8632 & 116.3 & 15.08 & 24.34 & 39.57 \\
0.8713 & 116.3 & 15.08 & 24.34 & 39.57 \\
0.8784 & 116.3 & 15.08 & 24.34 & 39.57 \\
0.8903 & 116.3 & 15.08 & 24.34 & 39.57 \\
0.8936 & 116.3 & 15.08 & 24.34 & 39.57 \\
0.9001 & 116.3 & 15.08 & 24.34 & 39.57 \\
0.9004 & 122.3 & 15.08 & 24.34 & 39.57 \\
0.9107 & 122.3 & 15.08 & 24.34 & 39.57 \\
0.9115 & 122.3 & 15.08 & 24.34 & 39.57 \\
0.9127 & 122.3 & 15.08 & 24.34 & 39.57 \\
0.92 & 122.3 & 15.08 & 24.34 & 39.57 \\
0.9215 & 122.3 & 15.08 & 24.34 & 39.57 \\
0.9299 & 122.3 & 15.08 & 24.34 & 39.57 \\
0.936 & 122.3 & 15.08 & 24.34 & 39.57 \\
0.9509 & 122.3 & 15.08 & 24.34 & 39.57 \\
0.965 & 122.3 & 15.08 & 24.34 & 39.57 \\
0.9837 & 122.3 & 15.08 & 24.34 & 39.57 \\
1.0013 & 122.3 & 15.08 & 24.34 & 39.57 \\
1.0116 & 124.3 & 15.08 & & 39.57 \\
\hline
\end{tabular}

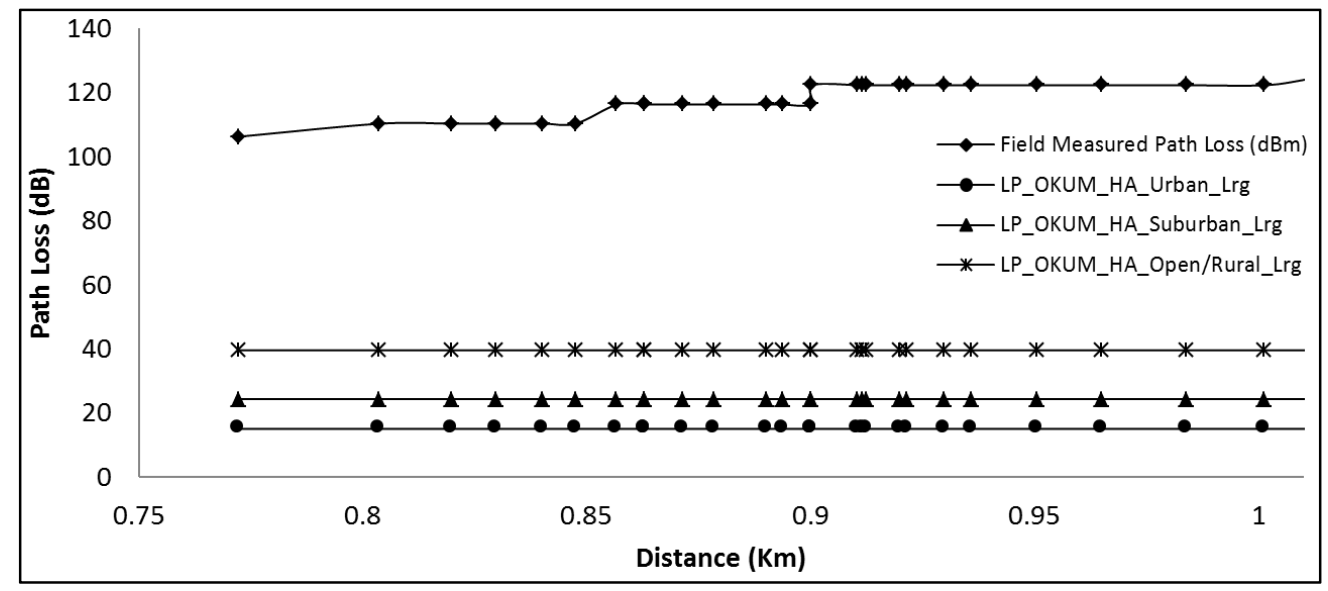

Figure 3. Path Loss vs Distance For The Untuned Hata-Okumura Models.

Table 3. Optimised Predicted Path Loss (dB) vs Distance For The Optimised HATA-OKUMURA Models.

\begin{tabular}{lllll}
\hline $\mathbf{d}(\mathbf{k m})$ & Field Measured Path Loss $\mathbf{( d B m})$ & Hata-Okumura Urban & Hata-Okumura Suburban & Hata-Okumura Open /RURAL AREA \\
\hline 0.7726 & 106.3 & 116.6 & 116.21 & 115.97 \\
0.8038 & 110.3 & 117.19 & 116.8 & 116.56 \\
0.8199 & 110.3 & 117.49 & 117.1 & 116.86 \\
0.8297 & 110.3 & 117.67 & 117.27 & 117.03 \\
0.8404 & 110.3 & 117.86 & 117.47 & 117.23 \\
0.8475 & 110.3 & 117.98 & 117.59 & 117.35 \\
0.8568 & 116.3 & 118.15 & 117.75 & 117.51 \\
0.863 & 116.3 & 118.25 & 117.86 & 117.62 \\
0.8632 & 116.3 & 118.26 & 117.86 & 117.63 \\
0.8713 & 116.3 & 118.4 & 118 & 117.77 \\
0.8784 & 116.3 & 118.52 & 118.13 & 117.89 \\
0.8903 & 116.3 & 118.72 & 118.33 & 118.09 \\
0.8936 & 116.3 & 118.77 & 118.38 & 118.14 \\
0.9001 & 116.3 & 118.88 & 118.49 & 118.25 \\
0.9004 & 122.3 & 118.89 & 118.5 & 118.26 \\
0.9107 & 122.3 & 119.06 & 118.67 & 118.43 \\
0.9115 & 122.3 & 119.07 & 118.68 & 118.44 \\
0.9127 & 122.3 & 119.09 & 118.7 & 118.46 \\
0.92 & 122.3 & 119.21 & 118.82 & 118.58 \\
0.9215 & 122.3 & 119.23 & 118.84 & 118.6 \\
0.9299 & 122.3 & 119.37 & 118.98 & 118.74 \\
\hline
\end{tabular}




\begin{tabular}{lllll}
\hline $\mathbf{d}(\mathbf{k m})$ & Field Measured Path Loss $\mathbf{( d B m )}$ & Hata-Okumura Urban & Hata-Okumura Suburban & Hata-Okumura Open /RURAL AREA \\
\hline 0.936 & 122.3 & 119.47 & 119.08 & 118.84 \\
0.9509 & 122.3 & 119.7 & 119.31 & 119.07 \\
0.965 & 122.3 & 119.92 & 119.53 & 119.29 \\
0.9837 & 122.3 & 120.21 & 119.82 & 119.58 \\
1.0013 & 122.3 & 120.47 & 120.08 & 119.84 \\
1.0116 & 124.3 & 120.63 & 120.24 & 120 \\
\hline
\end{tabular}

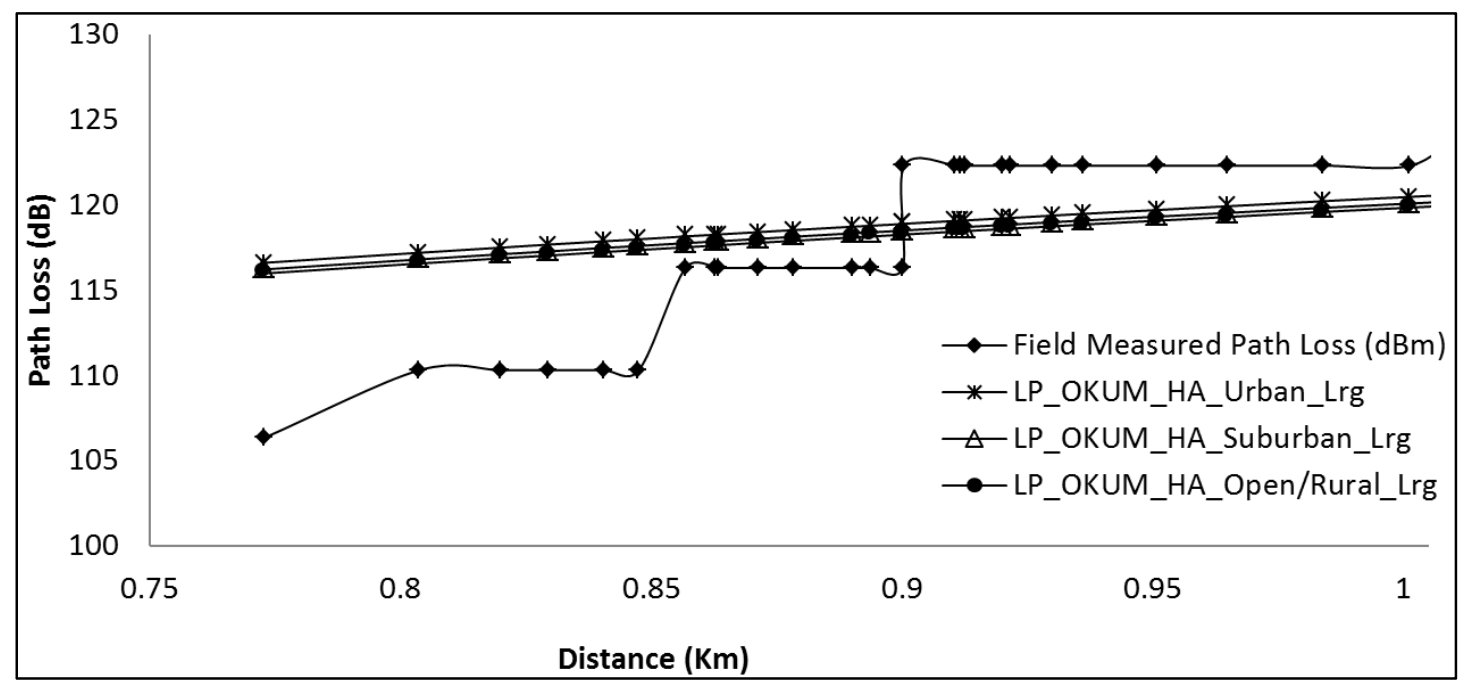

Figure 4. Path Loss vs Distance for the Optimized Hata-Okumura Models.

Table 4. Root Mean Square Error for the Hata-Okumura Models.

\begin{tabular}{llll}
\hline & $\begin{array}{l}\text { Root Mean Square Error (RMSE) } \\
\text { Hata-Okumura Urban }\end{array}$ & $\begin{array}{l}\text { Root Mean Square Error } \\
\text { Hata-Okumura Sub-Urban }\end{array}$ & $\begin{array}{l}\text { Root Mean Square Error Hata-Okumura } \\
\text { Rural/Open Area }\end{array}$ \\
\hline Untuned Models & 52.28 & 61.7 & 76.91 \\
Optimized Models & 13.54 & 13.44 & 13.34 \\
\hline
\end{tabular}

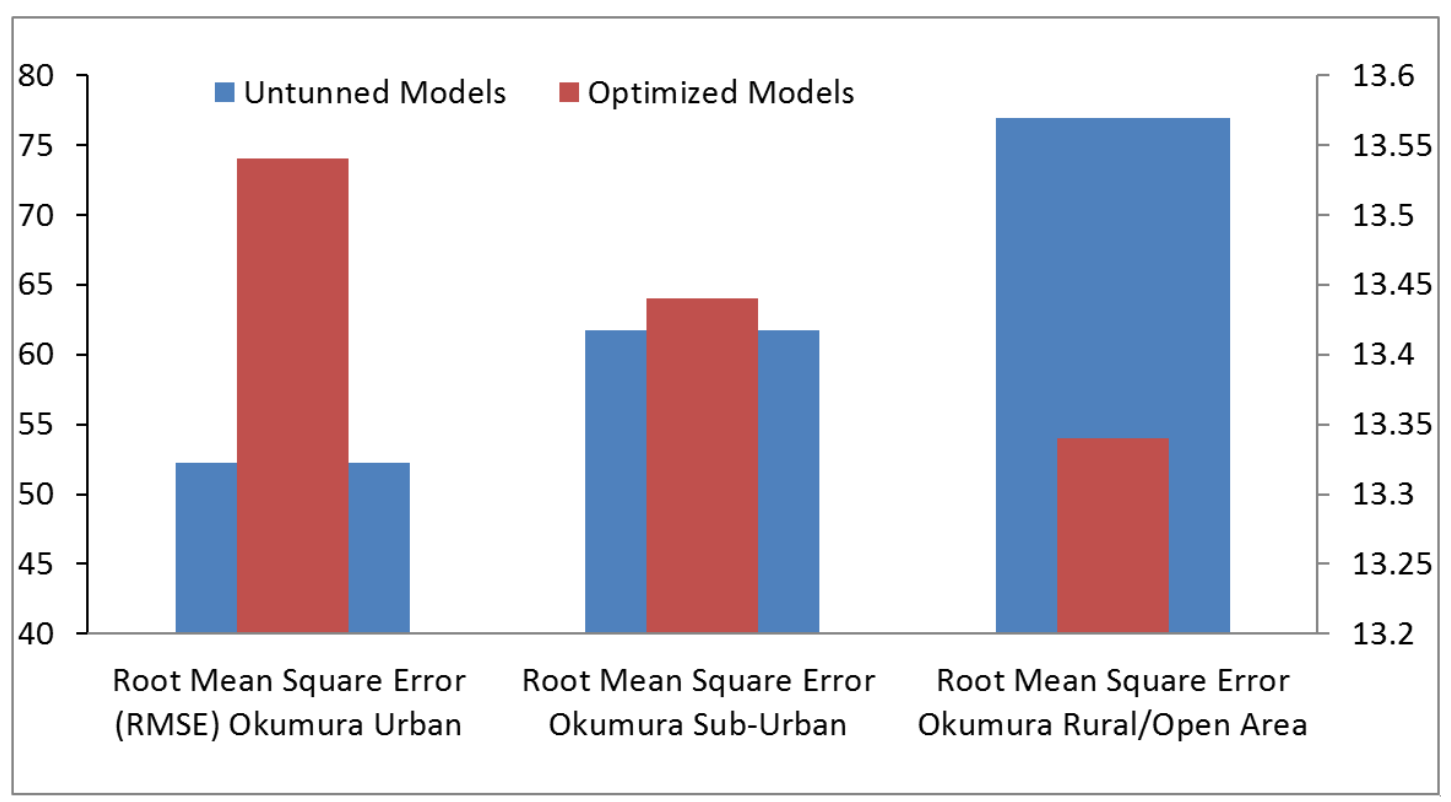

Figure 5. Bar chart Of The Root Mean Square Error For The Hata-Okumura Models.

Table 5. Mean Absolute Error (MAE) For The HATA-OKUMURA Models.

\begin{tabular}{llll}
\hline & $\begin{array}{l}\text { Mean Absolute Error (MAE) Okumura } \\
\text { Urban }\end{array}$ & $\begin{array}{l}\text { Mean Absolute Error Okumura } \\
\text { Sub-Urban }\end{array}$ & $\begin{array}{l}\text { Mean Absolute Error Okumura } \\
\text { Rural/Open Area }\end{array}$ \\
\hline Untuned Models & 50.77 & 60.43 & 75.9 \\
Optimized Models & 11.4 & 11.31 & 11.22 \\
\hline
\end{tabular}




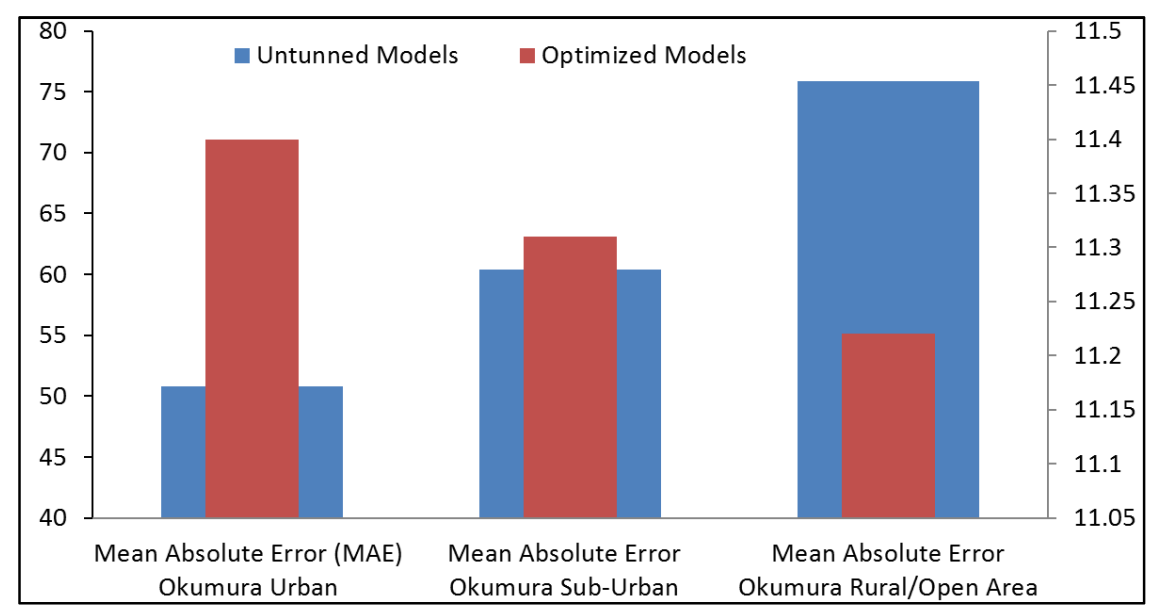

Figure 6. Mean Absolute Error (MAE) For The HATA-OKUMURA Models.

Table 6. Prediction Accuracy (\%) For The Models.

\begin{tabular}{llll}
\hline & $\begin{array}{l}\text { Prediction Accuracy (\%) Okumura } \\
\text { Urban }\end{array}$ & $\begin{array}{l}\text { Prediction Accuracy (5\%) Okumura } \\
\text { Sub-Urban }\end{array}$ & $\begin{array}{l}\text { Prediction Accuracy (5\%) Okumura } \\
\text { Rural/Open Area }\end{array}$ \\
\hline Untuned Models & 88.22 & 80.01 & 66.85 \\
Optimized Models & 96.71 & 96.74 & 96.76 \\
Improvement & 8.49 & 16.73 & 29.91 \\
\hline
\end{tabular}

\begin{tabular}{|c|c|c|c|}
\hline 100.00 & Untunned Models & Optimized Models & Г 96.76 \\
\hline 90.00 & & & 0675 \\
\hline 80.00 & & & 90.15 \\
\hline 70.00 & & & 96.74 \\
\hline 60.00 & & & 96.73 \\
\hline 50.00 & & & \\
\hline 40.00 & & & 96.72 \\
\hline 30.00 & & & 96.71 \\
\hline 20.00 & & & 96.70 \\
\hline 10.00 & & & \\
\hline 0.00 & $\begin{array}{l}\text { diction Accuracy (\%) } \\
\text { Okumura Urban }\end{array}$ & $\begin{array}{l}\text { liction Accuracy (5\%) } \\
\text { kumura Sub-Urban }\end{array}$ & 96.09 \\
\hline
\end{tabular}

Figure 7. Prediction Accuracy (\%) For The Models.

Where

The original Hata-Okumura model is given in Eq 21 to Eq 23 as:

For Urban Area

$$
L P_{O K_{-} H A T A(u r b a n)}=A+B * \log _{10}(d)
$$

For Suburban Area

$$
L P_{O K_{-} H A T A(\text { suburban })}=A+B * \log _{10}(d)-C
$$

For Open Area/Rural

$$
L P_{O K_{-} H A T A(o p e n / \text { rural })}=A+B * \log _{10}(d)-D
$$

The optimized model is given by adding an optimization constant, $K_{O P T}$ as follows;

For Urban Area

$$
L P_{O K_{-} H A T A(u r b a n)}=A+B * \log _{10}(d)+K_{O P T}
$$

\section{For Suburban Area}

$L P_{O K_{-} H A T A(s u b u r b a n)}=A+B * \log _{10}(d)-C+K_{O P T}$

For Open Area/Rural

$$
\begin{gathered}
L P_{O K_{-} H A T A(\text { open } / \text { rural })}=A+B * \log _{10}(d)-D+K_{O P T} \\
K_{O P T}=15.08 \text { for Urban } \\
K_{O P T}=24.34 \text { for Sub-Urban } \\
K_{O P T}=39.57 \text { for Rural/Open Area }
\end{gathered}
$$

\section{Conclusion}

A study on the three categories of Hata-Okumura pathloss model for urban, sub urban and rural/open area presented based on empirical measurements carried out for only $800 \mathrm{MHz}$ GSM network at Imo state University campus. The results showed that the Hata-Okumura model 
for the Urban area has the best prediction accuracy when not optimised but it has the lowest optimised prediction accuracy. On the other hand the Hata-Okumura model for the rural/open area has the least prediction accuracy when not optimised but it has the highest prediction accuracy when it is optimised. The results show that adoption of a model because it has the highest prediction accuracy is not the best approach to the selection of pathloss models. Rather the models should be optimised and the best optimised model should be adopted.

\section{References}

[1] Hanchinal C. S. and Muralidhara K. N. (2016) A Survey on the Atmospheric Effects on Radio Pathloss in Cellular Mobile Communication System. IJCST Vo 1. 7, Issue 1, Jan- March 2016.

[2] Rao, I. K., Donga, M., \& Chukka, M. (2013) Design and Study of Propagation Models in Wireless Communications (GSM) using Free Space Pathloss Model and Hata-Okumura Model with GUI. International Journal of Advanced Research in Electronics and Communication Engineering (IJARECE) Volume 2, Issue 12, December 2013

[3] Gupta, S. (2013, September). Comparative Pathloss Analysis Of Okumura And COST 231 Models For Wireless Mobile Communication Using MATLAB Simulation. In International Journal of Engineering Research and Technology (Vol. 2, No. 3 (March-2013)). ESRSA Publications.

[4] Alim, M. A., Rahman, M. M., Hossain, M. M., \& Nahid, A. A. (2010). Analysis of Large Scale Propagation Models for Mobile Communications in Urban Area. arXiv preprint arXiv: 1002. 2187.

[5] Sharma, P. K., \& Singh, R. K. (2012). Analysis of Large Scale Propagation Models \& RF Coverage Estimation. Analysis, 2 (2).

[6] Sati, G., \& Singh, S. A (2014) A review on outdoor propagation models in radio communication. International Journal of Computer Engineering \& Science, March 2014.

[7] Abhayawardhana, V. S., Wassell, I. J., Crosby, D., Sellars, M. P., \& Brown, M. G. (2005, May). Comparison of empirical propagation pathloss models for fixed wireless access systems. In Vehicular Technology Conference, 2005. VTC 2005-Spring. 2005 IEEE 61st (Vol. 1, pp. 73-77). IEEE.

[8] Kostanic, I., Guerra, I., Faour, N., Zec, J., \& Susanj, M. (2003).
Optimization and Application of WCY Lee Micro-cell Propagation Model in $850 \mathrm{MHz}$ Frequency Band. In Proceedings of Wireless Networking Symposium.

[9] Anderson H. R., (2003) "Fixed Broadband Wireless System Design: The Creation of Global Mobile Communications", John Wiley \& Sons, Inc., New York, NY, (2003).

[10] ANDERSON, H. R. (1997). Coverage Prediction for Digital Mobile Systems Part 1 [Online]. Available from: http://mrtmag.com/mag/radio_coverage_prediction digital2/index.html [Accessed on: 22/04/2006].

[11] Boggia, G., Camarda, P. and D'Alconzo, A, (2007) Modelling of Call Dropping in Well-Established Cellular Networks. EURASIP Journal on Wireles Communications and Networking. 2007: 1-11

[12] Deme, A., Dajab, D., Buba Bajoga, M. M. A., \& Choji, D. (2013). Hata-Okumura Model Computer Analysis for Path Loss Determination at $900 \mathrm{MHz}$ for Maiduguri, Nigeria. Mathematical Theory and Modeling, 3 (3), 1-9.

[13] Hata M., "Empirical formula for propagation loss in land mobile radio services," IEEE Transactions on Vehicular Technology, vol. vol. VT-29, September 1981.

[14] Neskovic, A., Neskovic, N., and Paunovic, G. (2000). Modern Approaches in Modeling of Mobile Radio Systems Propagation Environment. IEEE Commun. Surveys.

[15] Okumura, Y., Ohmori, E., Kawano, T., \& Fukuda, K. (1968). Field strength and its variability in VHF and UHF land-mobile radio service. Rev. Elec. Commun. Lab, 16 (9), 825-73.

[16] Ogbulezie, J. C., Onuu, M. U., Ushie, J. O., \& Usibe, B. E. (2013). Propagation models for GSM 900 and $1800 \mathrm{MHz}$ for Port Harcourt and Enugu, Nigeria. Network and Communication Technologies, 2 (2), 1.

[17] Obot, A., Simeon, O., \& Afolayan, J. (2011). Comparative analysis of path loss prediction models for urban macrocellular environments. Nigerian journal of technology, 30 (3), 50-59.

[18] Nadir, Z., \& Ahmad, M. I. (2010, March). Pathloss Determination Using Okumura-Hata Model And Cubic Regression For Missing Data For Oman. In Proceedings of the International MultiConference of Engineering and Computer scientist 2010 (Vol. 2).

[19] Singh, Y. (2012). Comparison of Okumura, Hata and COST-231 Models on the Basis of Path Loss and Signal Strength. International Journal of Computer Applications, 59 (11). pus, 8-9 April 2015. 